\title{
Landau damping of ion acoustic wave in Lorentzian multi-ion plasmas
}

\author{
Kashif Arshad, ${ }^{1}$ S. Mahmood, ${ }^{2,1, a)}$ and Arshad M. Mirza ${ }^{3}$ \\ ${ }^{1}$ National Center for Plasma Physics, Quaid-i-Azam University, Shadra Valley Road, Islamabad 44000, \\ Pakistan \\ ${ }^{2}$ Theoretical Plasma Physics Division (TPPD), PINSTECH, P.O. Nilore, Islamabad 44000, Pakistan \\ ${ }^{3}$ Department of Physics, Theoretical Plasma Physics Group, Quaid-i-Azam University, Islamabad 45320, \\ Pakistan
}

(Received 27 June 2011; accepted 12 August 2011; published online 29 September 2011)

The Landau damping rates of ion acoustic wave are studied by using Vlasov-Poisson model for unmagnetized Lorentzian or kappa distributed plasma containing electrons, positively and negatively charged ions. It is found that the damping rate of ion acoustic wave is increased with the decrease of kappa (i.e., the spectral index of Lorentzian distribution) value. The damping rates of the electrostatic wave in multi-ion component plasmas are discussed in detail which depends on electron to ion temperature ratio and ions masses and density ratios. The numerical results are also shown by choosing some typical experimental parameters of multi-ion plasmas. (C) 2011 American Institute of Physics. [doi:10.1063/1.3633237]

\section{INTRODUCTION}

The study of multi-ion plasma has gained interest due to its existence in space and laboratory plasmas. Space plasmas can contain the single ion species very rarely and mostly it consists of a combination of several ion species with different charges, degree of ionization, masses, and concentrations. $^{1,2}$ The multi-ion plasmas are studied theoretically as well as experimentally in different ways such as bi-ion plasmas, ${ }^{3-5}$ pure pair-ion plasmas, ${ }^{6-10}$ and pair-ion with electron plasmas. ${ }^{11,12}$ In order to study ion acoustic wave in negative ion plasma both $\mathrm{Ar} / \mathrm{SF}_{6}$ and $\mathrm{K} / \mathrm{SF}_{6}$ plasmas have been generated in $\mathrm{Q}$-machines by dc discharges. The sulfur hexaflouride $\left(\mathrm{SF}_{6}\right)$ gas readily produces negative ions in the plasma owing to its great electron affinity. The plasma produced was considered to be $\mathrm{Ar}^{+}-\mathrm{SF}_{6}^{-}, \mathrm{Ar}^{+}-\mathrm{F}^{-}$, and $\mathrm{Ar}^{+}-\mathrm{K}^{-}$ having mass ratios of $0.48,3.65$, and 3.74 respectively, with the presence of electrons as well. ${ }^{13-17}$ In the last few years, plasma containing significant concentration of negative ions with electrons and positive ions has gained interest and several authors have investigated the propagation of ionacoustic wave in such a multi-ion plasma. ${ }^{18-21}$ The plasma containing negative ions exists in the D-region of the ionosphere, ${ }^{22,23}$ in plasma processing reactors, ${ }^{24}$ and in neutral ion beam sources. ${ }^{25}$ Merlino and Loomis ${ }^{26}$ used $\left(\mathrm{Ar}^{+}, \mathrm{SF}_{6}^{-}\right)$ plasma for the experimental investigations of strong double layers in the laboratory experiments. The fullerene $\left(\mathrm{C}_{60}\right)$ pair-ion plasma has been produced successfully in Japan. ${ }^{6}$ Oohara et al. ${ }^{9}$ have produced hydrogen pair-ion plasma of lighter mass as compared to fullerene pair-ion plasmas in laboratory experiments. However, efforts are still going on to separate out electrons completely to produce pure hydrogen pair plasma on laboratory scale. In most of the study, the fluid theory has been used to study the ion acoustic wave in

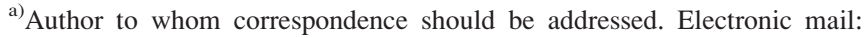
shahzadm100@gmail.com.
}

the presence of negative ions in plasmas and Maxwellian distribution of plasma particles has been assumed. In fact, non-Maxwellian velocity distributed plasmas have been observed in space and astrophysical plasma situations. The observed particles are found to have distribution of quasiMaxwellian up to mean thermal velocities with nonMaxwellian supra-thermal tails at high velocities or energies. The non-thermal plasmas are found to exist in the magnetospheres of the Earth, in planets, in the solar wind, etc. $^{27-35}$ Therefore, it will be interesting to study the Landau damping effect of the ion acoustic wave in the presence of negative ions for unmagnetized Lorentzian distributed multi-ion plasma.

The manuscript is organized in the following way; in Sec. II, we shall derive a new dispersion relation and estimate the damping rate of ion acoustic waves in a Lorentzian multi-ion plasmas using kinetic theory. The numerical results and discussion would be presented in Sec. III.

\section{DISPERSION RELATION AND LANDAU DAMPING RATE OF ION ACOUSTIC WAVE}

We are studying the electrostatic ion acoustic wave for non-Maxwellian distributed (generalized Lorentzian or kappa distribution) unmagnetized multi-ions and electron plasma. The Vlasov equation for the kinetic description of $\alpha$-species of charge $q_{\alpha}$ and mass $m_{\alpha}$ is given by

$$
\frac{\partial f_{\alpha}}{\partial t}+\mathbf{v} \cdot \nabla f_{\alpha}+\mathbf{a} \cdot \frac{\partial f_{\alpha}}{\partial \mathbf{v}}=0
$$

where $\alpha=+,-, e$ stands for the positive, negative ions, and electrons, respectively. For unmagnetized plasma, the acceleration of $\alpha$-particle can be expressed as $\mathbf{a}=\frac{q_{\alpha}}{m_{\alpha}} \mathbf{E}$. Here, we are considering electrostatic case for which $\mathrm{E}=-\nabla \phi$, where $\phi$ is the electrostatic potential. 
The linearized Vlasov equation can be written as

$$
\frac{\partial f_{\alpha_{1}}}{\partial t}+\mathbf{v} \cdot \nabla f_{\alpha_{1}}-\frac{q_{\alpha}}{m_{\alpha}} \nabla \phi_{1} \cdot \frac{\partial f_{\alpha_{0}}}{\partial \mathbf{v}}=0
$$

If we assume that the wave is propagating along the $z$-axis, then one can Fourier transform the above equation and can write the perturbed distribution function in the following way:

$$
f_{\alpha 1}=-\frac{q_{\alpha}}{m_{\alpha}} k_{\|} \phi_{1} \frac{\left(\frac{\partial f_{\alpha_{0}}}{\partial v_{\|}}\right)}{\left(\omega-v_{\|} k_{\|}\right)},
$$

where $k_{\|}=k_{z}$ in our notation, and for simplicity, we shall write $k$ instead of $k_{\|}$in the text. The Poisson equation in the linearized form can be written as

$$
\nabla^{2} \phi_{1}=-4 \pi \sum_{\alpha} q_{\alpha} n_{\alpha_{1}}
$$

where the perturbed number density of the $\alpha$ th species in terms of distribution function can be written as $n_{\alpha_{1}}=\iiint_{-\infty}^{+\infty} f_{\alpha 1} d^{3} v$. Now using the expression of $n_{\alpha_{1}}$ in Eq. (4) and after applying the Fourier transform, we get the following equation after some simplification:

$$
1=\sum_{\alpha} \frac{\omega_{p_{\alpha}}^{2}}{n_{\alpha_{0}} k^{2}} \iiint_{-\infty}^{+\infty} \frac{\frac{\partial f_{\alpha_{0}}}{\partial v_{\|}}}{\left(v_{\|}-\frac{\omega}{k}\right)} d^{3} v
$$

where $\omega_{p_{\alpha}}=\left(\frac{4 \pi n_{\alpha 0} e^{2}}{m_{\alpha}}\right)^{1 / 2}$ is the plasma frequency of the $\alpha$-species. In order to solve the above three dimensional velocity integral, we use cylindrical coordinates and obtain the following expression:

$$
1=\sum_{\alpha} \frac{\omega_{p_{\alpha}}^{2}}{n_{\alpha_{0}} k^{2}} \int_{0}^{2 \pi} d \phi \int_{0}^{\infty} v_{\perp} d v_{\perp} \int_{-\infty}^{\infty} \frac{\frac{\partial f_{\alpha_{0}}}{\partial v_{\|}}}{\left(v_{\|}-\frac{\omega}{k}\right)} d v_{\|} .
$$

The kappa distribution function for $\alpha$ species is given by $^{27,32-35}$

$$
f_{\alpha 0}=\frac{n_{\alpha_{0}}}{\pi^{\frac{3}{2}} \theta_{\perp_{\alpha}}^{2} \theta_{\|_{\alpha}}} \frac{\Gamma(\kappa+1)}{\kappa^{\frac{3}{2}} \Gamma\left(\kappa-\frac{1}{2}\right)}\left[1+\frac{v_{\|}^{2}}{\kappa \theta_{\|_{\alpha}}^{2}}+\frac{v_{\perp}^{2}}{\kappa \theta_{\perp_{\alpha}}^{2}}\right]^{-\kappa-1} .
$$

The Lorentzian thermal speed is related to the particle temperature (in the energy units) by the relation $\theta_{(\|, \perp)_{\alpha}}=\sqrt{\frac{2 \kappa-3}{\kappa}} v_{T_{(\|, \perp)_{\alpha}}}$, and thermal speed of the $\alpha$-species is defined as $v_{T_{\| \alpha, \perp \alpha}}=\sqrt{T_{\| \alpha, \perp \alpha} / m_{\alpha}}$. Here, $\kappa$ (kappa) is the spectral index and can have values $>3 / 2$ while $\Gamma$ is represented the standard Gamma function. The spectral index $\kappa$ is a measure of the slope of the energy spectrum of the supra-thermal particles in the tail of the velocity distribution function. ${ }^{17}$ The smaller is the value of $\kappa$ the more supra-thermal particles are in the distribution function tail and harder the energy spectrum. The kappa distribution approaches to Maxwellian as $\kappa \rightarrow \infty$.

Now using Eq. (7) in Eq. (6) and after performing the integration of the perpendicular velocity component, we obtain the following result:

$$
1=-\sum_{\alpha} \frac{\omega_{p_{\alpha}}^{2}}{k^{2}} \frac{2}{\pi^{\frac{1}{2}} \theta_{\|_{\alpha}}^{3}} \frac{\Gamma(\kappa+1)}{\kappa^{\frac{3}{2}} \Gamma\left(\kappa-\frac{1}{2}\right)} \int_{-\infty}^{\infty} \frac{v_{\|}}{\left(\frac{\omega}{k}-v_{\|}\right)}\left[1+\frac{v_{\|}^{2}}{\kappa \theta_{\|_{\alpha}}^{2}}\right]^{-\kappa-1} d v_{\|} .
$$

The above equation can be written in the form of dielectric function as follows:

$$
\epsilon(k, \omega)=1+\sum_{\alpha} \frac{\omega_{p_{\alpha}}^{2}}{k^{2}} \frac{2}{\theta_{\|_{\alpha}}^{3}} \frac{\Gamma(\kappa+1)}{\kappa^{\frac{3}{2}} \Gamma\left(\kappa-\frac{1}{2}\right)} Z\left(\frac{\omega}{k \theta_{\| \alpha}}\right) .
$$

Here, the plasma dispersion function for non-Maxwellian plasma is defined as

$$
Z\left(\frac{\omega}{k \theta_{\| \alpha}}\right)=\frac{1}{\sqrt{\pi}} \int_{-\infty}^{\infty} \frac{v_{\|}}{\left(\frac{\omega}{k}-v_{\|}\right)}\left[1+\frac{v_{\|}^{2}}{\kappa \theta_{\| \alpha}^{2}}\right]^{-\kappa-1} d v_{\|} .
$$

The dispersion function can be expanded in the limits described as follows, i.e., for $\left|\omega / k \theta_{\| \alpha}\right|<<1$, we have

$$
\begin{aligned}
Z\left(\frac{\omega}{k \theta_{\| \alpha}}\right)= & -\left(\theta_{\| \alpha} \kappa^{1 / 2} \frac{\lceil(\kappa+1 / 2)}{\lceil(\kappa+1)}\right) \\
& +i \sqrt{\pi}\left(\frac{\omega}{k}\right)\left[1+\frac{\omega^{2}}{\kappa k^{2} \theta_{\| \alpha}^{2}}\right]^{-\kappa-1},
\end{aligned}
$$

and for $\left|\omega / k \theta_{\| \alpha}\right|>>1$, we have

$$
\begin{aligned}
Z\left(\frac{\omega}{k \theta_{\| \alpha}}\right)= & -\theta_{\| \alpha} \kappa^{3 / 2} \frac{\lceil(\kappa-1 / 2)}{\lceil(\kappa+1)}\left(\frac{k \theta_{\| \alpha}}{\omega}\right)^{2} \\
& \times\left[1+\frac{3 \kappa}{(2 \kappa-3)}\left(\frac{k \theta_{\| \alpha}}{\omega}\right)^{2}\right]+\ldots \\
& +i \sqrt{\pi}\left(\frac{\omega}{k}\right)\left[1+\frac{\omega^{2}}{\kappa k^{2} \theta_{\| \alpha}^{2}}\right]^{-\kappa-1},
\end{aligned}
$$

where the integrals used are given as follows:

$$
\begin{aligned}
& \int_{-\infty}^{\infty}\left[1+\frac{v_{\|}^{2}}{\kappa \theta_{\| \alpha}^{2}}\right]^{-\kappa-1} d v_{z}=\sqrt{\pi} \theta_{\| \alpha} \kappa^{1 / 2} \frac{\Gamma\left(\kappa+\frac{1}{2}\right)}{\Gamma(\kappa+1)}, \\
& \int_{-\infty}^{\infty} v_{\|}^{2}\left[1+\frac{v_{\|}^{2}}{\kappa \theta_{\| \alpha}^{2}}\right]^{-\kappa-1} d v_{z}=\frac{\sqrt{\pi} \theta_{\| \alpha}^{3}}{2} \kappa^{3 / 2} \frac{\Gamma\left(\kappa-\frac{1}{2}\right)}{\Gamma(\kappa+1)}, \\
& \int_{-\infty}^{\infty} v_{\|}^{4}\left[1+\frac{v_{\|}^{2}}{\kappa \theta_{\| \alpha}^{2}}\right]^{-\kappa-1} d v_{z}=\frac{3 \sqrt{\pi} \theta_{\| \alpha}^{5}}{4} \kappa^{5 / 2} \frac{\Gamma\left(\kappa-\frac{3}{2}\right)}{\Gamma(\kappa+1)} .
\end{aligned}
$$

Using Eqs. (11) and (12) in Eq. (9), the dielectric function $\epsilon(k, \omega)$ for the ion acoustic wave in a non-Maxwellian multiion plasma under the limit $\left|\omega / k \theta_{\| e}\right|<<1$ for the electrons and for ions $\left|\omega / k \theta_{\| \pm}\right|>>1$ is obtained as follows: 


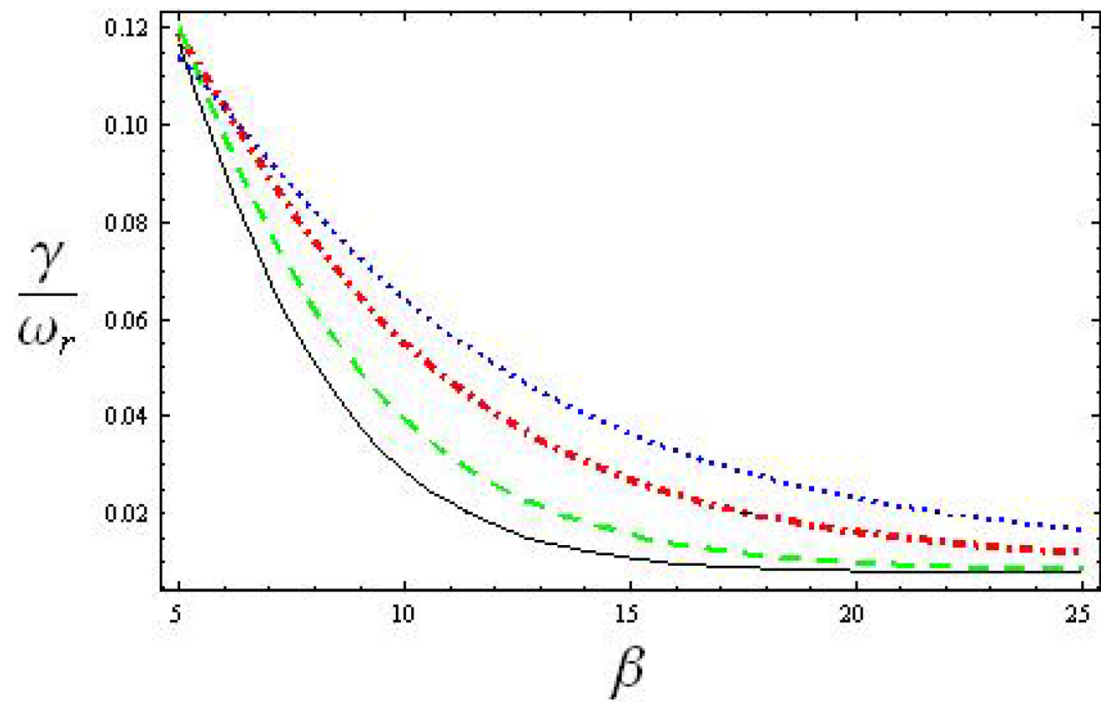

FIG. 1. (Color online) Normalized damping rates vs electron to ion temperature ratios are plotted for ion acoustic waves in two ion plasmas for different spectral indices $\kappa=5$ (blue dotted curve), 7 (red dotted-dashed curve), 15 (green dashed curve), 50 (black solid curve) for $H^{+}, H^{-}$, and electron plasmas.

$$
\begin{aligned}
\epsilon(k, \omega)= & \left\{2 \frac{\left(\kappa-\frac{1}{2}\right)}{\left(\kappa-\frac{3}{2}\right)}+k^{2} \lambda_{D e}^{2}\right\} \\
& -\frac{k^{2} \lambda_{D e}^{2} \omega_{p_{+}}^{2}}{\omega^{2}}\left[1+\frac{k^{2} \theta_{\|_{+}}^{2}}{\omega^{2}} \frac{3 \kappa}{2\left(\kappa-\frac{3}{2}\right)}\right] \\
& -\frac{k^{2} \lambda_{D e}^{2} \omega_{p_{-}}^{2}}{\omega^{2}}\left[1+\frac{k^{2} \theta_{\|_{-}}^{2}}{\omega^{2}} \frac{3 \kappa}{2\left(\kappa-\frac{3}{2}\right)}\right] \\
& +2 i \sqrt{\pi} \frac{\lambda_{D_{e}}^{2} \Gamma(\kappa+1)}{\kappa^{\frac{3}{2}} \Gamma\left(\kappa-\frac{1}{2}\right)}\left(\frac{\omega}{k}\right) \\
& \times\left[\frac{\omega_{p_{e}}^{2}}{\theta_{\|_{e}}^{3}}+\frac{\omega_{p_{+}}^{2}}{\theta_{\|_{+}}^{3}}\left\{1+\frac{\left(\frac{\omega}{k}\right)^{2}}{\kappa \theta_{\|_{+}}^{2}}\right\}^{-\kappa-1}\right. \\
& \left.+\frac{\omega_{p_{-}}^{2}}{\theta_{\|_{-}}^{3}}\left\{1+\frac{\left(\frac{\omega}{k}\right)^{2}}{\kappa \theta_{\|_{-}^{2}}^{2}}\right\}^{-\kappa-1}\right] .
\end{aligned}
$$

In order to obtain the Landau damping rate of ion acoustic wave in multi-ion plasma, we may let $\epsilon(\omega, k)=\epsilon_{r}(\omega, k)$ $+\epsilon_{i}(\omega, k)$, where $\epsilon_{r}(\omega, k)$ and $\epsilon_{i}(\omega, k)$ are the real and imagi-

nary parts of the dielectric response function, respectively. Also the wave frequency is given as $\omega=\omega_{r}+i \gamma$, where $\omega_{r}$ is the real part of frequency and $\gamma$ is the imaginary part of the frequency. It is also assumed that $\left|\epsilon_{r}(\omega, k)\right|<<\left|\epsilon_{i}(\omega, k)\right|$ and $\left|\omega_{r}\right|<<|\gamma|$. The real part of the dielectric function can be expressed as

$$
\begin{aligned}
\epsilon_{r}(\omega, k)= & \left(2 \frac{\left(\kappa-\frac{1}{2}\right)}{\left(\kappa-\frac{3}{2}\right)}+k^{2} \lambda_{D e}^{2}\right)-\frac{k^{2} \lambda_{D e}^{2} \omega_{p_{+}}^{2}}{\omega^{2}}\left[1+\frac{k^{2} \theta_{\|_{+}}^{2}}{\omega^{2}} \frac{3 \kappa}{2\left(\kappa-\frac{3}{2}\right)}\right] \\
& -\frac{k^{2} \lambda_{D e}^{2} \omega_{p_{-}}^{2}}{\omega^{2}}\left[1+\frac{k^{2} \theta_{\|_{-}}^{2}}{\omega^{2}} \frac{3 \kappa}{2\left(\kappa-\frac{3}{2}\right)}\right] .
\end{aligned}
$$

In order to obtain a dispersion relation, we put $\epsilon_{r}(\omega, k)$ equal to zero which gives

$$
\begin{aligned}
\omega_{r}^{2}= & \frac{k^{2} C_{s_{+}}^{2} N_{0}^{ \pm}}{\left[2 \frac{\left(\kappa-\frac{1}{2}\right)}{\left(\kappa-\frac{3}{2}\right)}+k^{2} \lambda_{D e}^{2}\right]}\left[1+\frac{3 \kappa}{2\left(\kappa-\frac{3}{2}\right)} \frac{k^{2} \theta_{\|_{+}}^{2}}{N_{0}^{ \pm} \omega_{r}^{2}}\left\{\left(\frac{n_{0_{+}}}{n_{0_{e}}}\right)\right.\right. \\
& \left.\left.+\left(\frac{n_{0_{-}}}{n_{0_{e}}}\right)\left(\frac{T_{-}}{T_{+}}\right)\left(\frac{m_{+}}{m_{-}}\right)^{2}\right\}\right],
\end{aligned}
$$

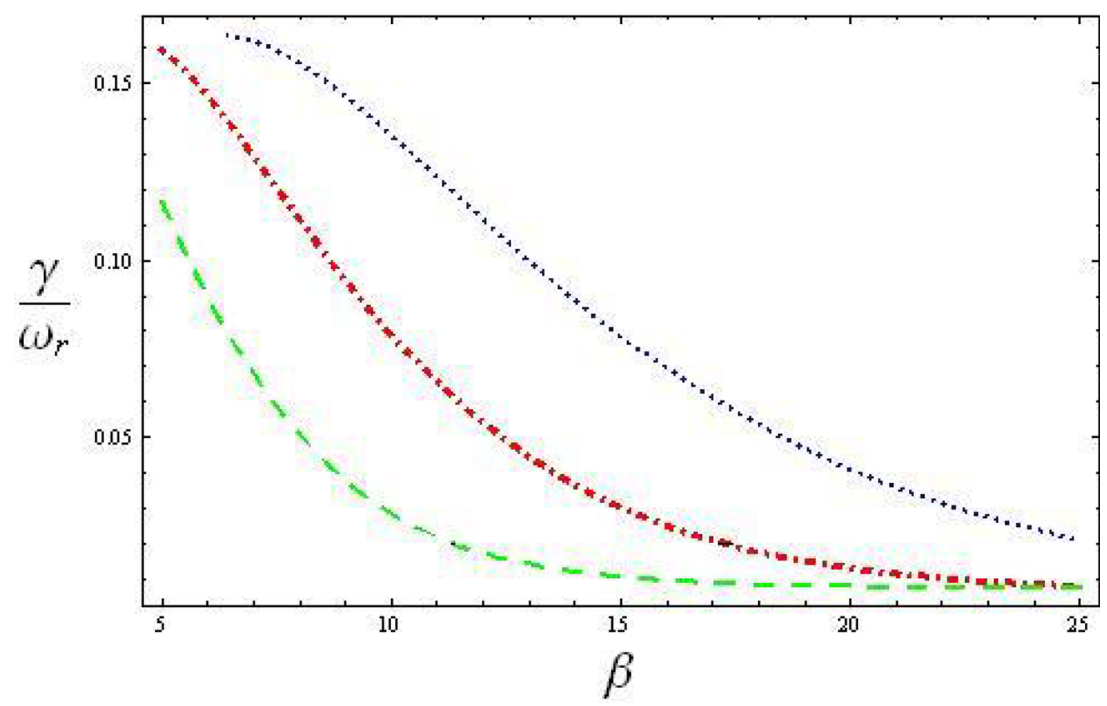

FIG. 2. (Color online) Normalized damping rates vs electron to ion temperature ratios are shown in the absence of negative ions, i.e., $n_{0} / n_{0_{e}}=0$ (blue dotted curve) and in the presence of negative ions, i.e., $0.2 / 0.8$ (red dotted-dashed curve), 0.4/0.6 (green dashed curve), respectively, for Maxwellian $(\kappa=50) \mathrm{H}^{+}, \mathrm{H}^{-}$ and electron plasmas. 


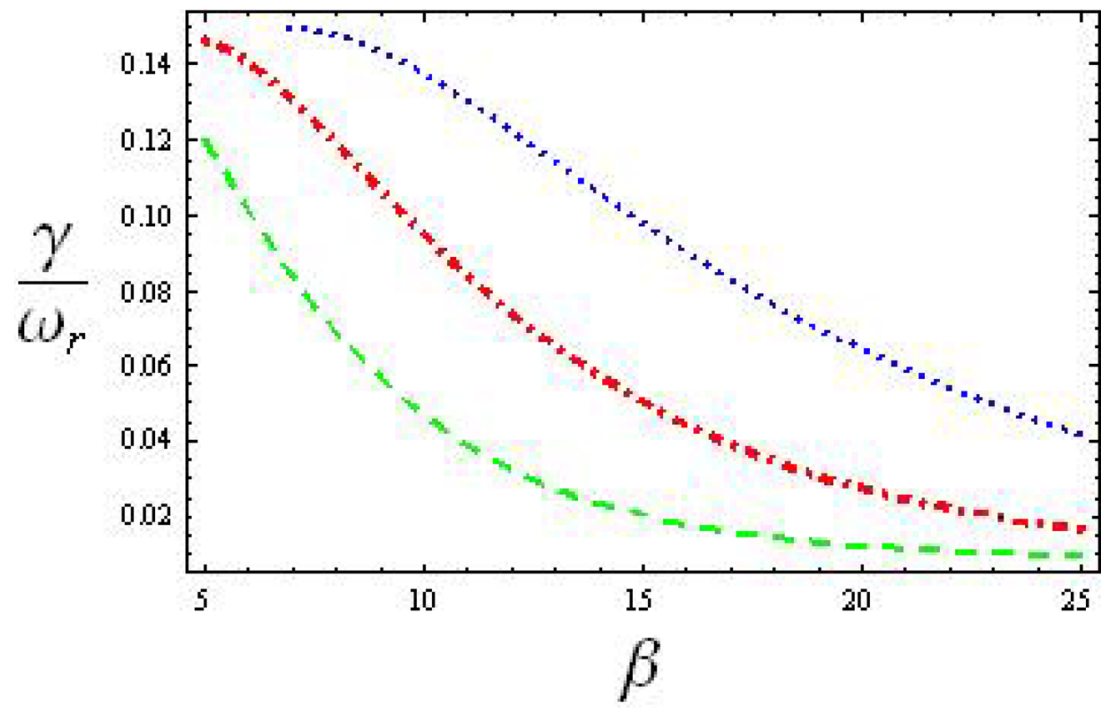

FIG. 3. (Color online) Normalized damping rates vs electron to ion temperature ratios are shown in the absence of negative ions, i.e., $n_{0_{-}} / n_{0_{e}}=0$ (blue dotted curve) and in the presence of negative ions, i.e., $0.2 / 0.8$ (red dotted-dashed curve), 0.4/0.6 (green dashed curve), respectively, for non-Maxwellian $(\kappa=10) \mathrm{H}^{+}$, $H^{-}$, and electron plasmas.

where the ion acoustic speed due to positive ions is $C_{S_{+}}$ $=\sqrt{T_{e} / m_{+}}$, electron Debye length is $\lambda_{D e}=\sqrt{T_{e} /\left(4 \pi n_{0 e} e^{2}\right)}$, and $N_{0}^{ \pm}=\left[n_{0_{+}} / n_{0_{e}}+n_{0_{-}} / n_{0_{e}}\left(m_{+} / m_{-}\right)\right]$has been defined.

The damping rate $\gamma$ is calculated from the real and imaginary parts of the dielectric function, i.e., $\gamma=-\epsilon_{i} /\left(\partial \epsilon_{r} / \partial \omega_{r}\right)$ which is given by

$$
\begin{aligned}
\gamma= & -\sqrt{\frac{\pi}{8}} \frac{\Gamma(\kappa+1)}{\left(\kappa-\frac{3}{2}\right)^{3 / 2} \Gamma\left(\kappa-\frac{1}{2}\right)^{ \pm}} \frac{\omega_{r}^{4}}{N_{0}^{ \pm} C_{S_{+}}^{2}}\left(\frac{m_{+}}{T_{+}}\right)^{1 / 2}\left[\left(\sqrt{\frac{m_{e} T_{+}}{m_{+} T_{e}}}\right)\right. \\
& +\left(\frac{n_{0_{+}}}{n_{0_{e}}}\right)\left(\frac{T_{e}}{T_{+}}\right)\left\{1+\frac{\left(\frac{\omega}{k}\right)^{2}}{\kappa \theta_{\|+}^{2}}\right\}^{-\kappa-1} \\
& \left.+\left(\frac{n_{0_{-}}}{n_{0_{e}}}\right)\left(\frac{T_{e}}{T_{-}}\right)\left(\sqrt{\frac{T_{+} m_{-}}{T_{-} m+}}\right) \times\left\{1+\frac{\left(\frac{\omega}{k}\right)^{2}}{\kappa \theta_{\|-}^{2}}\right\}^{-\kappa-1}\right] .
\end{aligned}
$$$$
\frac{\gamma}{\omega_{r}}=-\sqrt{\frac{\pi}{8}} \frac{\Gamma(\kappa+1)}{\left(\kappa-\frac{3}{2}\right)^{3 / 2} \Gamma\left(\kappa-\frac{1}{2}\right)} \frac{\sqrt{N_{0}^{ \pm}}}{\left(2 \frac{\left(\kappa-\frac{1}{2}\right)}{\left(\kappa-\frac{3}{2}\right)}+k^{2} \lambda_{D_{e}}^{2}\right)^{3 / 2}}\left[\sqrt{\frac{m_{e}}{m_{+}}}\right.
$$$$
+\left(\frac{n_{0_{+}}}{n_{0_{e}}}\right)\left(\frac{T_{e}}{T_{+}}\right)^{3 / 2}\left[1+\frac{C_{s_{+}}^{2} N_{0}^{ \pm}}{\kappa \theta_{\|_{+}}^{2}\left(2 \frac{\left(\kappa-\frac{1}{2}\right)}{\left(\kappa-\frac{3}{2}\right)}+k^{2} \lambda_{D_{e}}^{2}\right)}\right.
$$$$
\left.+\frac{3}{(2 \kappa-3) N_{0}^{ \pm}}\left(\left(\frac{n_{0_{+}}}{n_{0_{e}}}\right)+\left(\frac{n_{0_{-}}}{n_{0_{e}}}\right)\left(\frac{T_{-}}{T_{+}}\right)\left(\frac{m_{+}}{m_{-}}\right)^{2}\right)\right]^{-\kappa-1}
$$$$
+\left(\frac{n_{0_{-}}}{n_{0_{e}}}\right)\left(\frac{T_{e}}{T_{-}}\right)^{3 / 2} \sqrt{\frac{m_{-}}{m+}}\left[1+\frac{C_{s_{+}}^{2} N_{0}^{ \pm}}{\kappa \theta_{\|_{-}}^{2}\left(2 \frac{\left(\kappa-\frac{1}{2}\right)}{\left(\kappa-\frac{3}{2}\right)}+k^{2} \lambda_{D_{e}}^{2}\right)}\right.
$$$$
+\frac{3}{(2 \kappa-3) N_{0}^{ \pm}} \frac{\theta_{\|_{+}}^{2}}{\theta_{\|_{-}}^{2}}
$$$$
\left.\left.\times\left(\left(\frac{n_{0_{+}}}{n_{0_{e}}}\right)+\left(\frac{n_{0_{-}}}{n_{0_{e}}}\right)\left(\frac{T_{-}}{T_{+}}\right)\left(\frac{m_{+}}{m_{-}}\right)^{2}\right)\right]^{-\kappa-1}\right] .
$$

The normalized damping rate of ion acoustic wave in multiion Lorentzian plasma can be written as

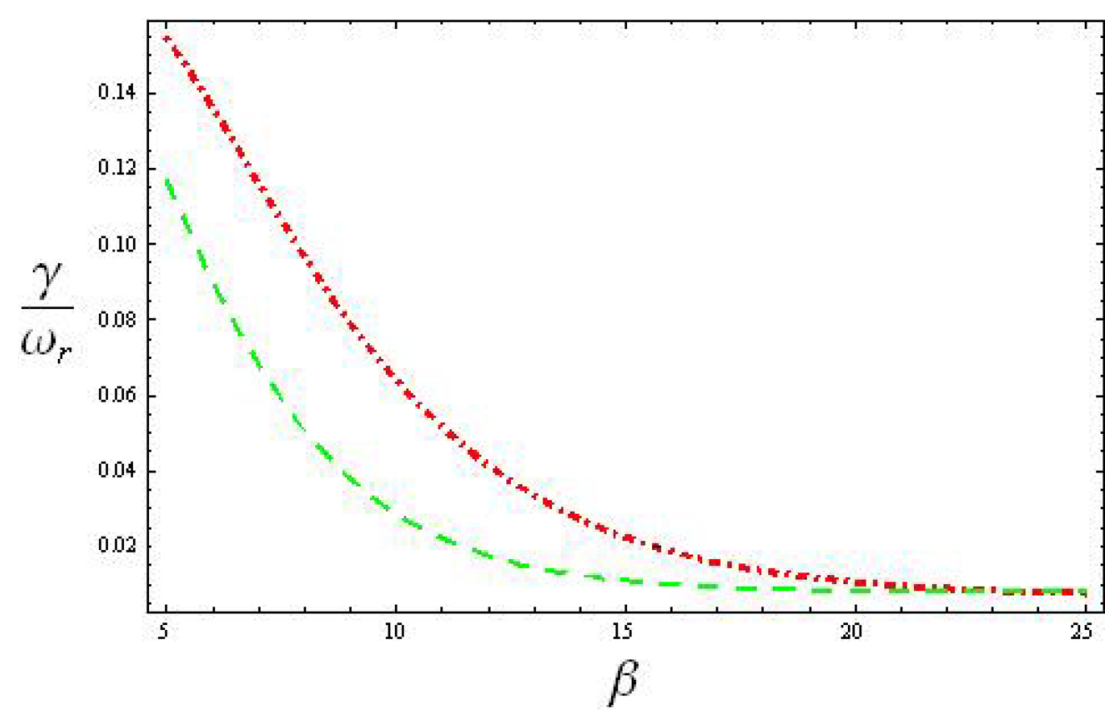

FIG. 4. (Color online) Normalized damping rates vs electron to ion temperature ratios are shown for Maxwellian plasma at $\kappa=50$. The damping curves correspond to hydrogen-oxygen $\left(\mathrm{H}^{+}-\mathrm{O}_{2}^{-}\right.$electron) plasma (red dotted-dashed curve), hydrogen-hydrogen $\left(H^{+}-H^{-}\right.$ electron) plasma (green dashed curve), respectively, and are plotted for fixed density ratio $n_{0-} / n_{0_{e}}=0.4 / 0.6$. 


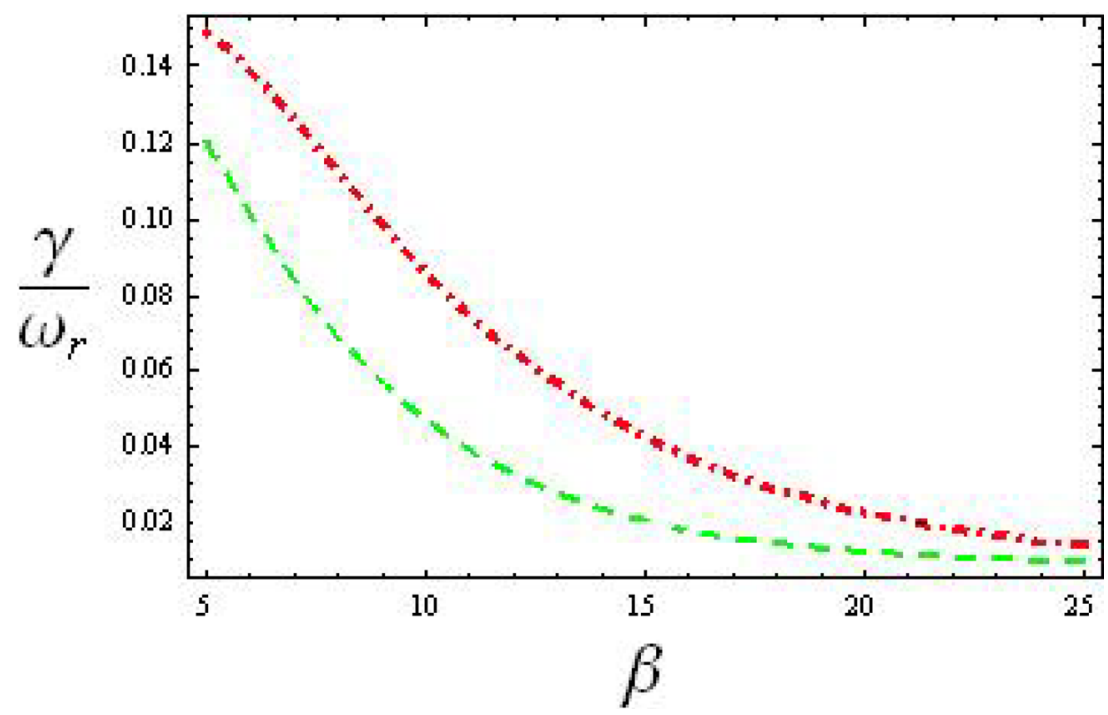

FIG. 5. (Color online) Normalized damping rates vs electron to ion temperature ratios are shown for nonMaxwellian Lorentzian plasmas at $\kappa=10$. The damping curves correspond to hydrogen-oxygen $\left(\mathrm{H}^{+}-\mathrm{O}_{2}^{-}\right.$ electron) plasma (red dotted-dashed curve), hydrogenhydrogen $\left(H^{+}-H^{-}\right.$electron) plasma (green dashed curve), respectively, and are plotted for density ratio $n_{0_{-}} / n_{0_{e}}=0.4 / 0.6$.

In the long wavelength limit, $k^{2} \lambda_{D_{e}}^{2}<<1$, and considering the same ion temperatures but different masses of positive and negative ions, i.e., $T_{+}=T_{-}=T_{i}$ and defining a parameter $\beta=T_{e} / T_{i}$ which represents the ratio of electron to ion temperature, i.e., $i=+,-$ for positive and negative ions, respectively. The normalized damping rate of the ion acoustic wave in terms of $\beta$ for multi-ion plasma is given by

$$
\begin{aligned}
\frac{\gamma}{\omega_{r}}= & -\sqrt{\frac{\pi}{8}} \frac{\Gamma(\kappa+1)}{\Gamma\left(\kappa-\frac{1}{2}\right)} \frac{\sqrt{N_{0}^{ \pm}}}{2^{3 / 2}\left(\kappa-\frac{1}{2}\right)^{3 / 2}}\left[\sqrt{\frac{m_{e}}{m_{+}}}\right. \\
& +\left(\frac{n_{0_{+}}}{n_{0_{e}}}\right) \beta^{3 / 2}\left[1+\frac{\beta N_{0}^{ \pm}}{2(2 \kappa-1)}+\frac{3}{(2 \kappa-3) N_{0}^{ \pm}} \delta\right]^{-\kappa-1} \\
& +\left(\frac{n_{0_{-}}}{n_{0_{e}}}\right) \beta^{3 / 2} \sqrt{\frac{m_{-}}{m_{+}}}\left[1+\frac{\beta N_{0}^{ \pm}}{2(2 \kappa-1)}\right. \\
& \left.\left.+\frac{3}{(2 \kappa-3) N_{0}^{ \pm}} \frac{m_{-}}{m_{+}} \delta\right]^{-\kappa-1}\right]
\end{aligned}
$$

where $\delta=\left\{\left(\frac{n_{0_{+}}}{n_{0_{e}}}\right)+\left(\frac{n_{0_{-}}}{n_{0_{e}}}\right)\left(\frac{m_{+}}{m_{-}}\right)^{2}\right\}$. For large values of $\kappa \rightarrow \infty$, our results reduce to the well known Maxwellian distributed multi-ion plasmas, ${ }^{11}$ whereas for finite value of $\kappa$, new effects appear in Lorentzian multi-ion plasma case. The magnitude of normalized damping rates $\left|\frac{\gamma}{\omega_{r}}\right|$ vs. $\beta\left(=T_{e} / T_{i}\right)$ of ion acoustic wave as described by Eq. (17) for Lorentzian multi-ion component plasma are plotted in Sec. III. Equation (17) is valid only to study the weak damping effects in the system.

\section{NUMERICAL RESULTS AND DISCUSSION}

In this section, the numerical results are presented by taking some typical values of multi-ion plasma such as typical densities of negative and positive ions, as well as the temperature of two ions and electrons from laboratory experiments. $^{17,21,22}$ The normalized damping rates of ion

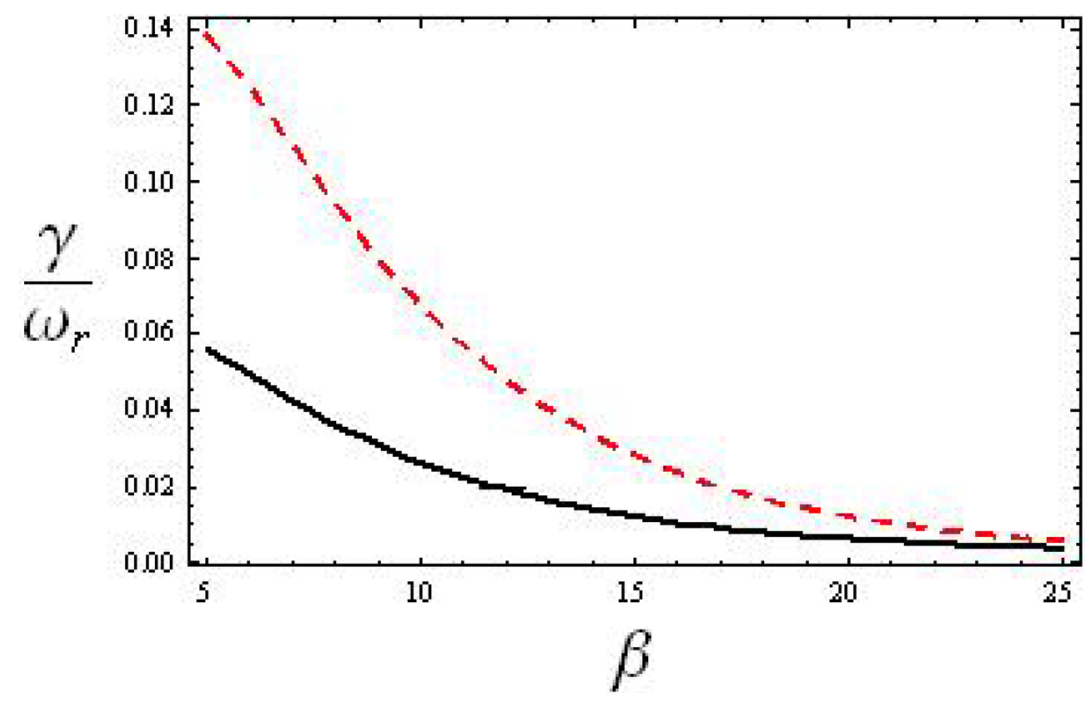

FIG. 6. (Color online) Normalized damping rates vs electron to ion temperature ratios are shown for Lorentzian plasma at $\kappa=10$. The damping curves correspond to argon-flourine $\left(\mathrm{Ar}^{+}-\mathrm{F}^{-}\right.$electron) plasma (black solid curve), argon-sulfurhexaflouride $\left(\mathrm{Ar}^{+}-\mathrm{SF}_{6}^{-}\right.$ electron) plasma (red dotted curve), respectively, and are plotted for density ratio $n_{0_{-}} / n_{0_{e}}=0.4 / 0.6$. 


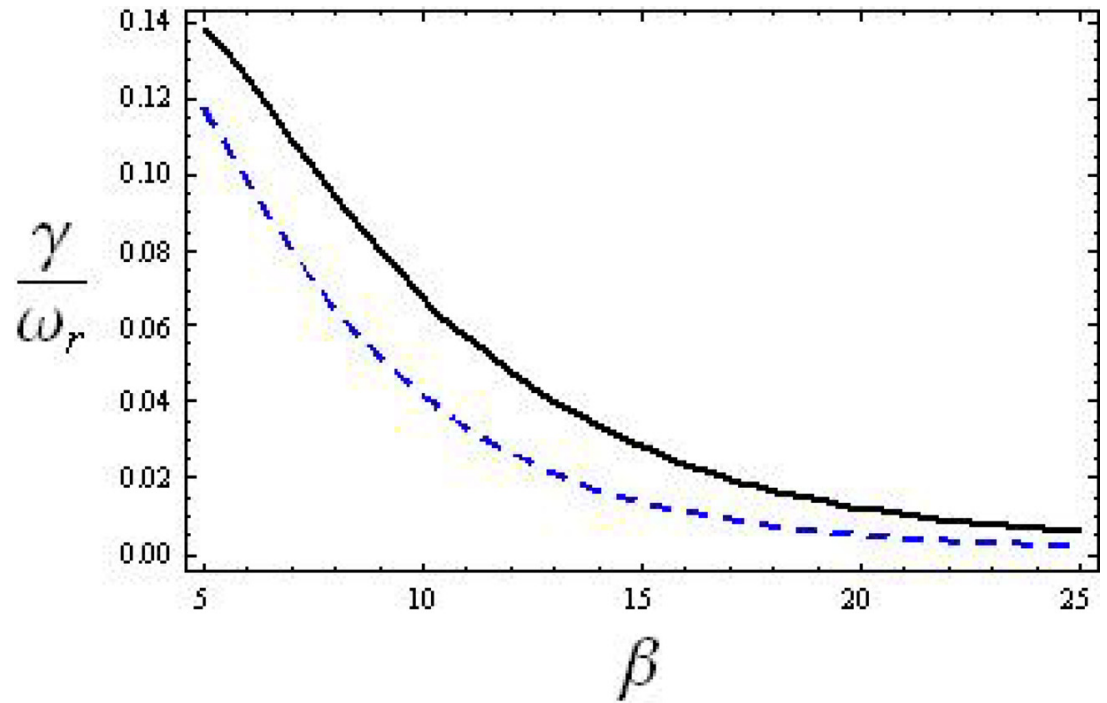

FIG. 7. (Color online) Normalized damping rates vs electron to ion temperature ratios are shown at fixed value of $\kappa=10$. The damping curves correspond to argon-sulfurhexaflouride $\left(A r^{+}-S F_{6}^{-}\right.$electron) plasma (black solid curve), argon-sulfurhexaflouride $\left(X_{e}^{+}-S F_{6}^{-}\right.$ electron) plasma (blue dotted curve), respectively, and are plotted for density ratio $n_{0_{-}} / n_{0_{e}}=0.4 / 0.6$. acoustic wave in multi-ion plasma are plotted against the temperature ratio of electron to the ion in two-ion plasmas which contain positive and negative ion densities $n_{0+} \sim 10^{10} \mathrm{~cm}^{-3}$ and $n_{0-}=0.4 \times 10^{10} \mathrm{~cm}^{-3}$, respectively. The damping rates of the electrostatic wave in two-ion hydrogen plasma at different Kappa values are shown in Figure 1. It is clear from figure that the damping rate of the ion acoustic wave is increased with the decrease in the kappa value. The decrease in the kappa value increases the energetic particles in the tail of the energy spectrum and there will be more energetic particles for wave-particle interaction to increase the Landau damping effect of the ion acoustic wave for Lorentzian multi-ion plasmas. The decrease in the damping rate of ion acoustic wave in hydrogen plasma with electrons in the presence and absence of negative hydrogen ions are shown for Maxwellian and Lorentzian distributed plasma in Figs. 2 and 3 , respectively. It is evident from the figures that the damping rate of the ion acoustic wave is decreased in the presence of negative hydrogen ions in hydrogen-pair plasma in the presence of electrons. The damping rates for same mass of positive ions, i.e., $\left(\mathrm{H}^{+}-\mathrm{H}^{-}-\mathrm{e}\right)$ and $\left(\mathrm{H}^{+}-\mathrm{O}_{2}^{-}-\mathrm{e}\right)$ plasmas are plotted for same density ratio of ions for Maxwellian and Lorentzian plasma cases which are shown in Figs. 4 and 5, respectively. It is evident from figures that in case of heavier mass of negative ions, i.e., $\mathrm{O}_{2}^{-}$in comparison with lighter mass of negative ions, i.e., $\mathrm{H}^{-}$, the damping rate of ion acoustic wave is found to be increased. The increase in the damping rate of the ion acoustic wave for Lorentzian distributed $\left(\mathrm{Ar}^{+}-\mathrm{F}^{-}-\mathrm{e}\right)$ and $\left(\mathrm{Ar}^{+}-\mathrm{SF}_{6}^{-}-\mathrm{e}\right)$ plasmas are shown in Fig. 6 with the same density ratio of negative ions to electrons. The damping rate of the ion acoustic wave is shown in Fig. 7 by varying the mass of positive ions. It is evident from figure that the damping rate of the wave is decreased by increasing the mass of positive ions, i.e., argonsulfurhexaflouride $\left(\mathrm{Ar}^{+}-\mathrm{SF}_{6}^{-}-\mathrm{e}\right)$, xenon-sulfurhexaflouride $\left(\mathrm{X}_{e}^{+}-\mathrm{SF}_{6}^{-}-\mathrm{e}\right)$ in multi-ion plasma. The variation in the damping rates of the ion acoustic waves for different spectral indices at different values of $\beta$ and wave number $k$ are plotted and shown in Fig. 8.
To summarize, we have studied the Landau damping effects of ion acoustic wave in unmagnetized Lorentzian distributed multi-ion component plasmas. It is found that the damping rate of the electrostatic wave is increased with the decrease in the value of kappa, i.e., the harder the energy spectrum increases the damping rate of the wave. However, in the presence of negative ions in multiion plasmas, the damping rate of the ion acoustic wave is found to be decreased. Therefore, ion acoustic wave can easily be excited in the presence of negative ions in comparison with the absence of negative ions in multi-ion plasmas. The mass of both positive and negative ions also plays an important role for the excitation of the ion acoustic wave in multi-ion plasmas. Our findings are general and may be applicable to explain some aspects of non-Maxwellian multi-ion plasmas which can exist in space and laboratory experiments.

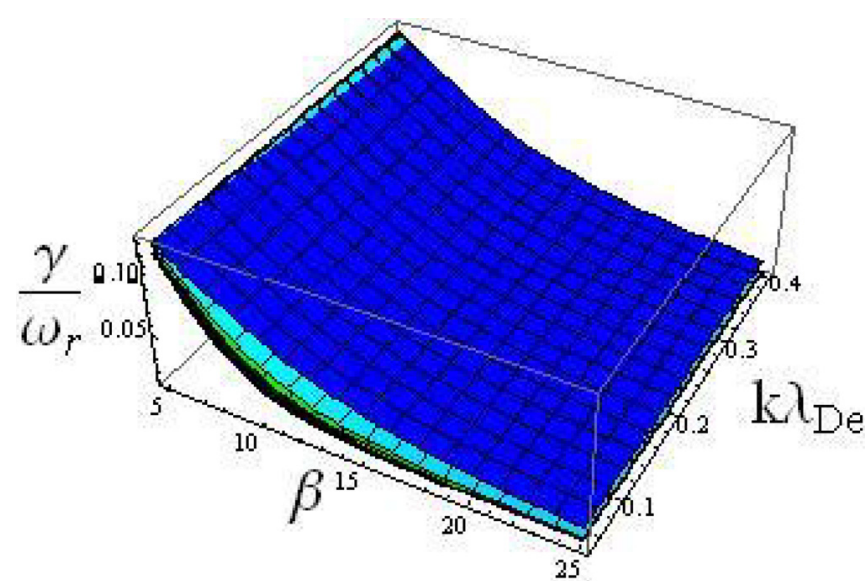

FIG. 8. (Color online) Normalized damping rates are plotted for ion acoustic plasma waves in $H^{+}, H^{-}$, and electron plasmas for different spectral indices $\kappa=5$ (upper (blue) surface), 10 (first below (sky blue) surface), 15 (second below (green) surface), 50 (lowest below (black) surface) are shown against the ratio of electron to ion temperature, i.e., $\beta=5$ to 25 and normalized wave number $k$ varies from 0.05 to 0.4 at density ratio $n_{0_{-}} / n_{0_{e}}=0.4 / 0.6$. 


\section{ACKNOWLEDGMENTS}

This research work was partially supported by the Quiadi-Azam University Research Fund, URF-Project (2011).

${ }^{1}$ G. Mann, P. Hackenberg, and E. Marsch, J. Plasma Phys. 58, 205 (1997).

${ }^{2}$ I. Lourakis, F. Verheest, and N. F. Cramer, Phys. Plasmas 14, 022306 (2007).

${ }^{3}$ E. Dubinin and K. Sauer, AstroPhys. Space Sci. 264, 273 (2006).

${ }^{4}$ L. Yang and D. J. Wu, Plasma Phys. Controlled Fusion 49, 119 (2007).

${ }^{5}$ J. F. Zhang, Y.-Y. Wang, and L. Wu, Phys Plasmas 16, 062102 (2009).

${ }^{6}$ W. Oohara and R. Hatakeyama, Phys. Rev. Lett. 91, 205005 (2003).

${ }^{7}$ W. Oohara and O. Fukumasa, Rev. Sci. Instrum. 81, 023507 (2010).

${ }^{8}$ W. Oohara, D. Date, and R. Hatakeyama, Phys. Rev. Lett. 95, 175003 (2005).

${ }^{9}$ W. Oohara and R. Hatakeyama, Phys. Plasmas 14, 055704 (2007).

${ }^{10}$ W. Oohara and R. Hatakeyama, Phys. Rev. Lett. 91, 205005 (2003).

${ }^{11}$ H. Saleem, Phys. Plasmas 14, 014505 (2007).

${ }^{12}$ S. Mahmood, H. Ur-Rehman and H. Saleem, Phys. Scr. 80, 035502 (2009).

${ }^{13}$ M. Rosenberg and R. L. Merlino, Planet Space Sci. 55, 1464 (2007).

${ }^{14}$ Y. Nakamura, T. Odagiri, and I. Tsukabayashi, Plasma Phys.Controlled Fusion 39, 105 (1997).

${ }^{15}$ Y. Nakamura, H. Bailung, and K. E. Lonngren, Phys. Plasmas 6, 3466 (1999).

${ }^{16}$ W. F. El, N. A. El-Bedwehy, and E. F. El. Shamy, Phys. Plasmas 18, 033703 (2011).

${ }^{17}$ N. Sato, Plasma Sources Sci. Technol. 3, 395 (1994).
${ }^{18}$ S. Mahmood and H. Saleem, J. Geophys. Res. 110, A09306, doi:10.1029/ 2004JA010758 (2005).

${ }^{19}$ N. D’ Angelo and R. L. Merlino, Planet Space Sci. 44, 239 (1996).

${ }^{20}$ R. Ichiki, M. Shindo, S. Yoshimura, T. Watanabe, and Y. Kawai, Phys. Plasmas 8, 4275 (2001).

${ }^{21}$ S. Qian, W. Lotko, and M. K. Hudson, J. Geophys. Res. 94, 1339 (1989).

${ }^{22}$ S. G. Tagare and R. V. Reddy, Plasma Phys.Controlled Fusion 29, 671 (1987).

${ }^{23}$ H. Massey, Negative Ions (Cambridge University Press, Cambridge, 1976), p. 663.

${ }^{24}$ R. A. Gottscho and C. E. Gaebe, IEEE Trans. Plasma Sci. 14, 92 (1986).

${ }^{25}$ M. Bacal and G. W. Hamilton, Phys Rev. Lett. 42, 1538 (1979).

${ }^{26}$ R. L. Merlino and J. J. Loomis, Phys. Fluids B 2, 2865 (1990).

${ }^{27}$ T. K. Baluku, M. A. Helberg, I. Kourakis, and N. S. Siani, Phys. Plasmas 17, 053702 (2010).

${ }^{28}$ T. K. Baluku and M. A. Hellberg, Phys. Plasmas 15, 123705 (2008).

${ }^{29}$ S. P. Christon, D. G. Mitchell, D. J. Williams, L. A. Frank, C. Y. Huang, and T. E. Eastman, J. Geophys. Res. 93, 2562 (1988).

${ }^{30}$ M. Maksimovic, V. Pierrard, and J. F. Lemaire, Astron. Astrophys. 324, 725 (1997).

${ }^{31}$ V. Pierrard, H. Lamy, and J. Lemarie, J. Geophys. Res. 109, A02118, doi:10.1029/2003JA010069 (2004).

${ }^{32}$ K. Arshad and S. Mahmood, Phys. Plasmas 17, 124501 (2010).

${ }^{33}$ N. Rubab, N. V. Erkaev, and H. K. Biernat, Phys. Plasmas 16, 103704 (2009).

${ }^{34}$ S. Zaheer, G. Murtaza, and H. A. Shah, Phys. Plasmas 11, 5 (2004).

${ }^{35}$ M.-J. Lee, Phys. Plasmas 14, 032112 (2007). 